\title{
Leadership's Guide for Achieving Sustainable Lean Six Sigma Program Change in Higher Educational Institutions
}

\author{
Sandra Furterer, Ph.D, MBA \\ Email: sfurterer1@udayton.edu \\ Department of Engineering Management, Systems, and Technology, University of Dayton
}

\begin{abstract}
Purpose: This paper will highlight the current research, practices and an innovative framework for enhancing Lean Six Sigma leadership engagement, and program success in higher educational institutions.

Methodology/Approach: Based on the literature and the author's experience in industry, the author has developed a framework that Lean Six Sigma leaders in higher educational institutions can utilize to guide program success and implement an infrastructure for governance that helps to ensure alignment of the improvement initiatives.

Findings: Three key themes are found in the research literature that contribute to successful process organizational change including: 1) need for executive sponsorship's active role in defining the infrastructure for change; 2) development of purposeful program governance; and 3) importance of aligning the program's initiatives with the organization's strategic plan. The critical framework elements that are addressed within the framework are executive sponsorship, program governance, strategic and cultural alignment.

Practical Implications: Higher Educational Institutions can apply the framework to enhance the success of the Lean Six Sigma programs and ensure alignment to their key strategic initiatives.
\end{abstract}

Keywords: Lean Six Sigma, leadership, organizational change, process architecture

Paper Type: Conference 


\section{Introduction}

Performing Lean Six Sigma projects without an infrastructure beneath the projects is like building a building or a bridge without an architecture or blueprint - it does not ensure success. Three key themes found in the research literature that contribute to successful process organizational change include: 1) need for executive sponsorship's active role in defining the infrastructure for change; 2) development of purposeful program governance; and 3) importance of aligning the program's initiatives with the organization's strategic plan. The executive sponsorship element of the framework includes ensuring that leadership understands and enacts the leadership role, and develops and incorporates a program communication strategy. The program governance includes developing the program structure for governing the program, including defining the roles and responsibilities of the program's resources, developing the program's mission and key messages. The strategic and cultural alignment includes applying a process architecture to ensure selection and alignment of process improvement initiatives to the organization's strategic plan and core processes that support the customers' needs, and ensures alignment and development of a plan to align the organization's culture with the Lean Six Sigma principles.

\subsection{Literature Review}

The author researched the quality and operational excellence literature to understand the factors that have been found to ensure successful operational change. There has been a consensus across many articles for the need for top management commitment to an operational excellence program, regardless of the tools or methodologies applied (Barry, 2008), (Catherwood, 2005), (Goh, 20011), (Brady and Allen, 2006). There is a need for senior leaders to actively participate on projects as a champion, removing barriers and challenges to change (Barry, 2008), (Catherwood, 2005), (Hahn, 2005). Senior management needs to demonstrate that Six Sigma is the engine of the organization's operational excellence transformation strategy (Catherwood, 2005). Senior leadership needs to create the infrastructure for the operational excellence program (Catherwood, 2005), (Hahn, 2005). Top management also needs to provide the strategic alignment between project goals and the needs of the business (Catherwood, 2005), (Sloan, 2001), (Montgomery, 2013).

\section{Methodology and Framework}

Based on the literature and the author's experience in industry, the author has developed a framework that Lean Six Sigma leaders in higher educational institutions can utilize to guide program success and implement an infrastructure for governance that helps to ensure alignment of the improvement initiatives.

The three foundational elements of the Six Sigma Leadership Engagement Framework (S-SLEF) are:

1) Sponsorship

2) Governance

3) Strategic alignment

A graphic in figure 1 summarizes the Six Sigma Leadership Engagement Framework (S-SLEF) 


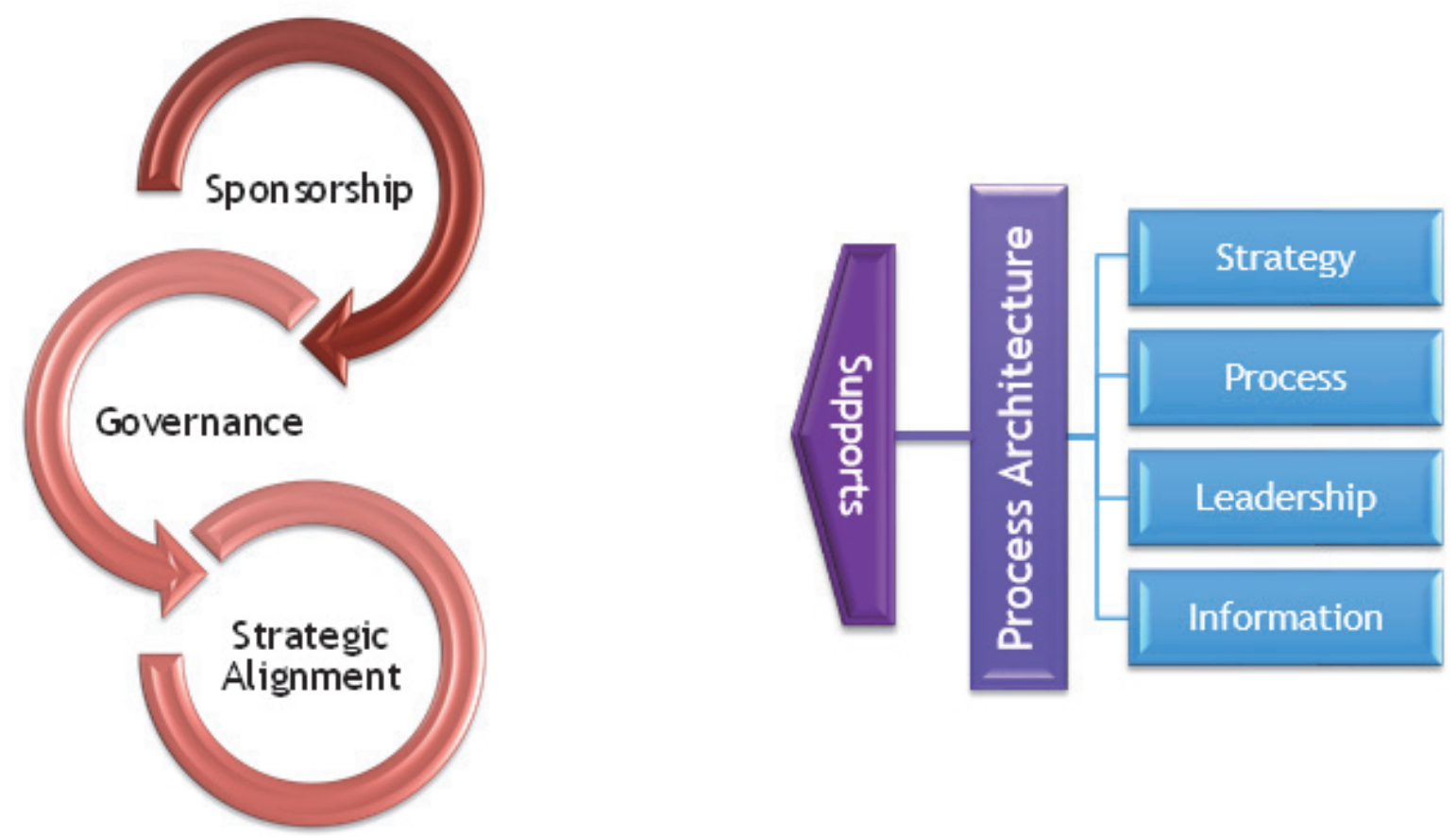

Figure 1. Six Sigma Leadership Engagement Framework (S-SELF)

\section{Results - Sponsorship}

The senior leadership in the higher educational institution should support operational excellence. It is critical for them to develop the program infrastructure and governance for the operational excellence program. They need to make the decision of what the program is called, what are the guiding principles, and what methodology and tools are used. They need to define the name of the program-- is it "Lean Six Sigma", "Operational Excellence", "Process Transformation", or some other name that describes the leaders' vision for operational excellence and process improvement. The author has designed and deployed multiple Lean Six Sigma programs in varying industries, such as healthcare, retail, financial services and higher education. Each culture is different, as well as their core values. Healthcare organizations tend to like to name their improvement program to demonstration excellence, as well as indicate it is an organizationwide project, using a name such as Enterprise Excellence. A large international retail organization used the term Global Continuous Improvement to demonstrate the global and ongoing improvement nature of their program. A particular financial services organization named their program, Process Transformation, to communicate the need to transform their processes. It is the leaders' responsibility to think through the name of the program, so that it conveys the goal of the program. Leadership also needs to ensure strategic alignment of the program and project initiatives. They should remove barriers to change, which always exist as a natural component of systems and organizational change. It is equally important to reward, recognize and celebrate success. It is senior leadership's role to communicate a consistent message to the institution and its stakeholders, internal and external to the institution. An example of a vision for a process transformation program from the author's experience is (Furterer, 2015): 
Process Transformation will help make processes simpler, faster and more valuable.

Simpler $=$ Less steps, less complexity, easier to perform, less waste and cost

Faster $=$ More timely to meet customers' expectations

Valuable $=$ Meeting customers' requirements at low cost and high service

The leadership communication should happen in one-on-one conversations, department, college or school, university-wide and to the outside world. Senior leadership should also ensure that appropriate resources are assigned to manage the program and participate in the projects.

Senior leadership should also ensure cultural alignment. Colleges and universities are steeped in bureaucracy, tradition, pomp and circumstance. They all have a unique culture that is important to understand and align the operational excellence program to the culture. An exercise that can help assess alignment between the cultural values of the institution and the Lean Six Sigma principles is shown in figure 2 . The leaders can be asked to identify which of the cultural values align to the Lean Six Sigma principles, to ensure that the leaders see that there is alignment, or where there is a gap to be closed. Many times these cultural values can be derived from the organization's strategic plan and sometimes are displayed on the organization's website. If these values have not formally been written down, a planning exercise can be performed with stakeholders within the organization to brainstorm and prioritize the core values of the organization. With higher educational organizations existing or sometimes 50 to 100 years or more, most HEIs have well-established cultures and core values to which they align their strategies, goals and initiatives.

\begin{tabular}{|l|l|}
\hline \multicolumn{2}{|c|}{ Cultural Values } \\
\hline Research excellence
\end{tabular}

Figure 2. Cultural Alignment Exercise 


\subsection{Governance}

Many alternative governance structures could be equally successful. The important point is to define a governance structure. The author has effectively used the structure shown in figure 3 in multiple organizations, one in the financial services sector and the other in a hospital system (Furterer, 2009),

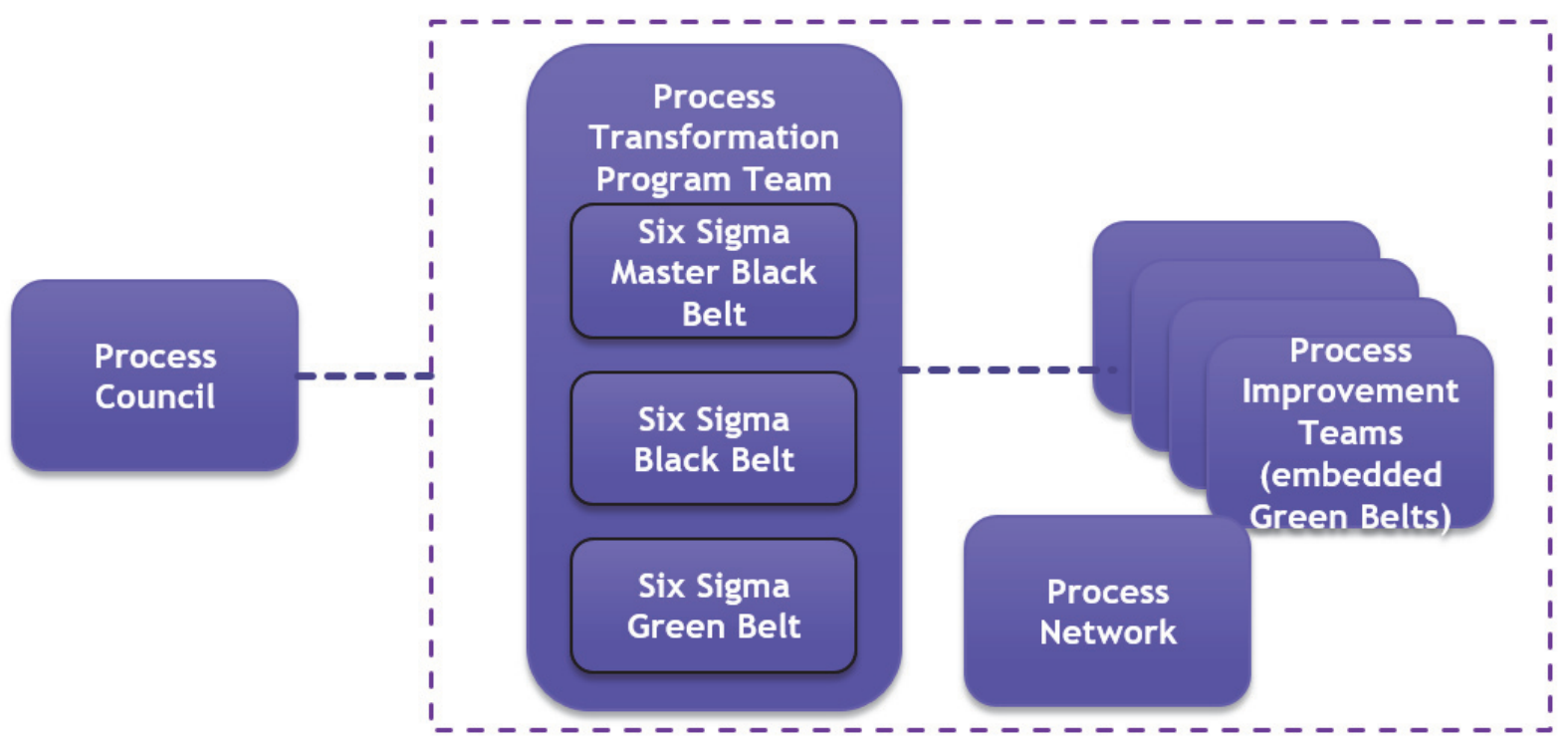

Figure 3. Lean Six Sigma Program Governance Structure

The program governance structure consists of a process council, process improvement teams and a process transformation program team. The overarching program process council consists of key leaders across the institution that perform the following activities:

- Identify and prioritize process transformation strategy and initiatives

- Monitor process transformation projects' status, reporting and progress

- Reinforce a culture of continuous improvement

The process improvement teams have embedded certified Lean Six Sigma Green Belts that are mentored by Process Transformation Black Belts to improve processes based upon the Lean Six Sigma DMAIC (Define-Measure-Analyze-Improve-Control) methodology and tools. The process improvement teams' responsibilities are to:

- Design and implement improvements

- Measure and monitor on-going process metrics and improvements

The process transformation program team is a dedicated team that runs the process transformation program and interacts with the process council for strategic direction and mentors process improvement teams. The process transformation program team's core responsibilities are to: 
- Develop and deliver the Lean Six Sigma training and certification program

- Organize project initiatives

- Track project status and report to process council

- Mentor project teams, Green Belts and Black Belts

\subsection{Strategic Alignment}

Process architecture can be used to ensure that the Lean Six Sigma program and project initiatives are aligned to the strategies of the organization. While Enterprise Architecture "relates to understanding the universe of the distinct elements that comprise the enterprise and how these elements interrelate" (Minole, 2008), the process architecture focuses on understanding the elements related to processes. The author developed a Strategic Business Process Architecture (SBPA) (Furterer, 2009) that includes the following conceptual meta models:

- Strategies

- Processes

- Leadership

- Information

The high-level view of the SBPA models are shown in figure 4.

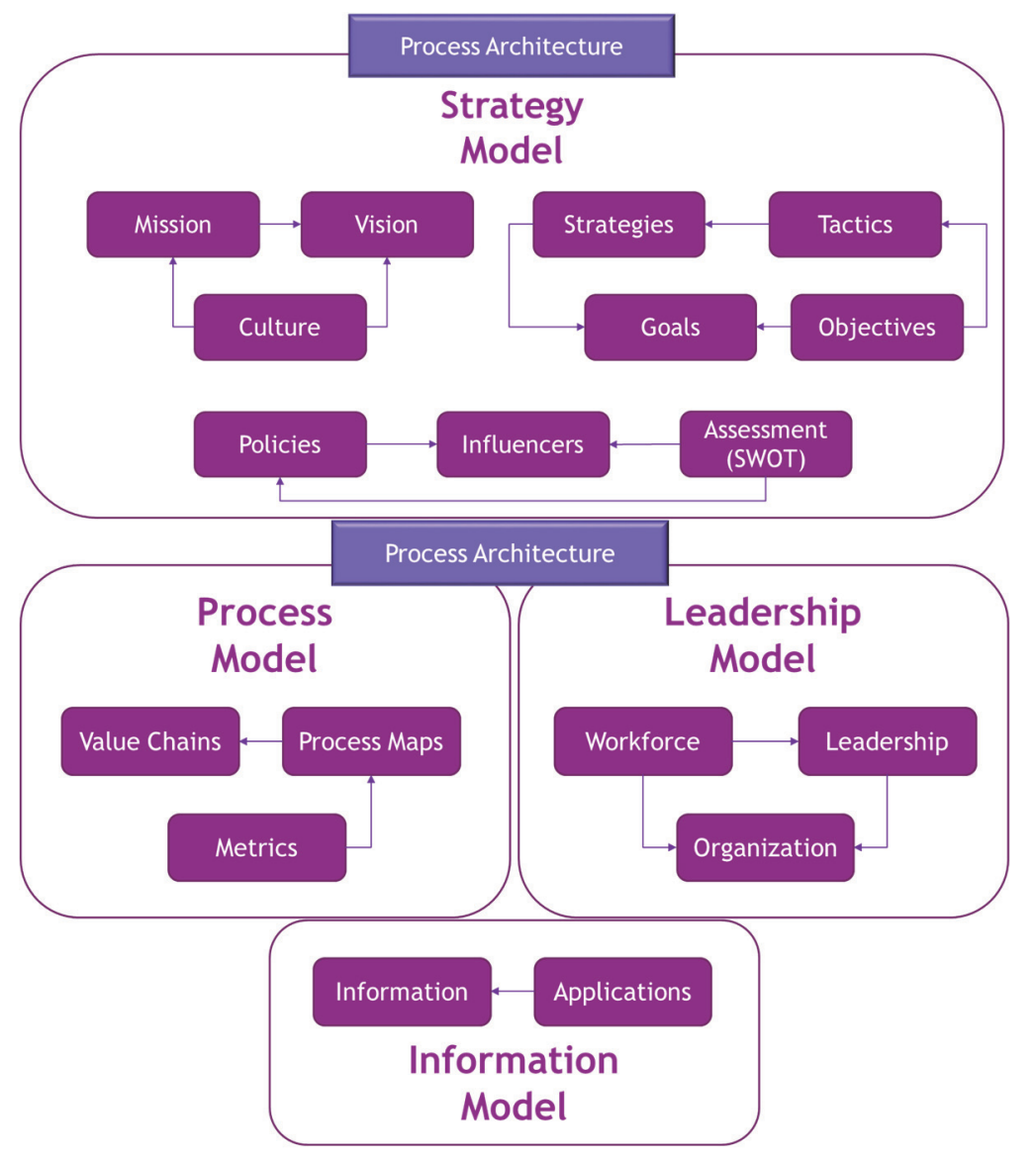

Figure 4. High-Level View of the Strategic Business Process Architecture Meta Models 
It is critical that these models are populated through strategic planning and process design and improvement sessions, within the institution to ensure alignment between the enterprise, operational and performance levels of the enterprise. The enterprise level consists of the strategic plan, including the strategies, objectives, tactics, and process improvement projects. The operational level incorporates the value chains, functions, processes and activities and their relationships to each other. The performance level includes the Critical to Satisfaction criteria, metrics and results. Each of these models are related to each other in a series of interrelated matrices. The strength of relationship between each of the models and their elements are defined within the matrices. This provides a prioritization of the project initiatives that are related to the strategic plan. It also provides a decomposition of the value chains, functions, processes, and activities that make up the process architecture. The Critical to Satisfaction criteria which describe the elements that are important to the customers' satisfaction, and are related to the metrics and results. The matrices are shown in figure 5 .

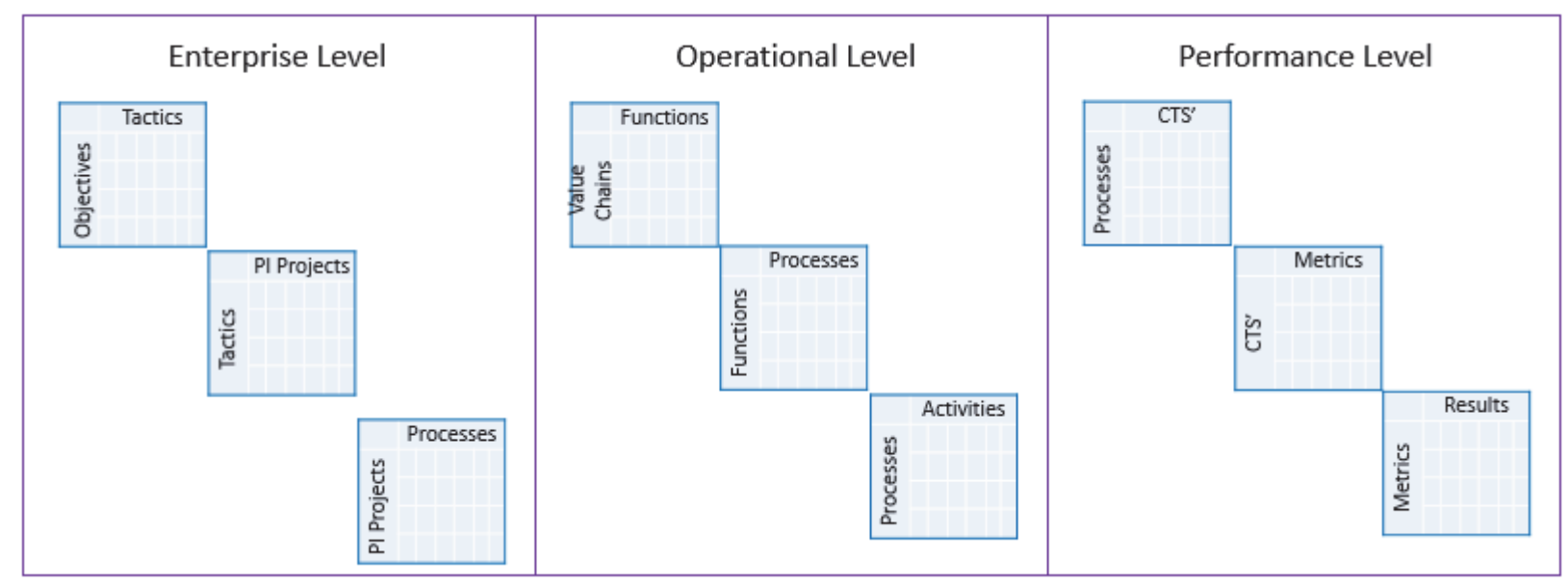

Figure 5. Strategic Alignment Matrices

\section{Conclusions}

The Six Sigma Strategic Leadership Engagement Framework (S-SLEF) provides alignment between the organization's strategies and their Lean Six Sigma program and projects. The framework provides the following benefits:

- Supports the key drivers identified in the literature

- Keeps the organization focused on what is important to make the Six Sigma program successful

- Maintains consistent messaging and communication

- Ensures cultural alignment and movement towards the continuous improvement principles

- Provides visibility to results and success

- Enables rewards and celebration

The framework provided has been applied in a financial services organization and a hospital system (Furterer, 2009), and has been effective in guiding the strategic alignment with the core business processes through the S-SLEF framework. 


\section{Future Work}

The author has identified and facilitated Lean Six Sigma projects through experiential learning opportunities in a Six Sigma course at several universities. These projects helped the institutions gain an awareness of how Lean Six Sigma methods and tools could enhance operational excellence in a university setting. However, the full framework needs to be executed and tested in a higher educational institution to ensure that there are no factors unique to these environments that would preclude it from being effective.

\section{References}

Barry, Eoin, SIX SIGMA - SO WHAT, WHO CARES, WHY ME?, Engineers Journal, Volume 62: Issue 5, June 2008.

Brady, James E., and Allen, Theodore, T., Six Sigma Literature: A Review and Agenda for Future Research, Quality and Reliability Engineering International, 2006, 22: 335-367.

Catherwood, P., Champions of the Cause, IEE, Manufacturing Engineer, October/ November 2005, PP $40-43$.

Furterer, Sandy, Enabling Enterprise Six Sigma through Business Process Architecture Modeling Techniques, Quality Management Forum, Fall 2009.

Furterer, Sandra, Enterprise Performance Excellence in a Hospital: A Framework, Methodology and Case Study, Operational Excellence Conference, October 2009.

Furterer, Sandra, Lean Six Sigma Leadership Guide for Program Success: S-SLF Framework, Ohio State University Lean Six Sigma Solutions Breakfast, May 2015.

Goh, T.N., Six Sigma in Industry: Some Observations After 25 Years, Quality and Reliability Engineering International, 2011, 27, PP 221-227.

Hahn, G.J., Six Sigma: 20 Key Lessons Quality and Reliability Engineering International, 2005; $21: 225-233$

Minoli, Daniel, Enterprise Architecture A to Z: Frameworks, Business Process Modeling, SOA, and Infrastructure Technology, CRC Press, Boca Raton, 2008.

Montgomery, Doug, Lean Six Sigma and Quality Management, Editorial, Quality and Reliability Engineering International; 2013, 29: page 935.

Sloan, Kelly, The Path to a Sustainable Playbook, Industrial Engineer, April 2001, PP 42-46. 